\title{
Thyroid Dysfunction in Ménière's Disease: A Comprehensive Review
}

\author{
Gloria Hwang $^{\mathrm{a}}$ Robert Saadi $^{\mathrm{b}}$ Vijay A. Patel ${ }^{\mathrm{b}}$ Jeffrey Liaw ${ }^{\mathrm{b}}$ Huseyin Isildak ${ }^{\mathrm{b}}$ \\ ${ }^{a}$ College of Medicine, The Pennsylvania State University, Hershey, PA, USA; b Department of Otolaryngology, Head \\ and Neck Surgery, College of Medicine, The Pennsylvania State University, Hershey, PA, USA
}

\section{Keywords}

Autoimmune · Hypothyroidism · Ménière's disease ·

Otology · Sensorineural hearing loss · Thyroid · Update

\begin{abstract}
Introduction: The precise etiology of Ménière's disease (MD) remains unknown; however, given the association of MD with serum antibodies and human leukocyte antigen (HLA) complex, several studies have proposed a relationship between MD and thyroid disorders. Similarly, multiple hypotheses exist regarding the metabolic disturbances of fluctuating thyroid hormone as a potential contributing agent in the development of MD. Methods: A total of 171 abstracts were identified and screened by 2 independent reviewers. Based on inclusion and exclusion criteria, 8 studies were selected for final analysis. Due to heterogeneity of clinical data, metaanalysis was not feasible. Results: The prevalence of autoimmune thyroid disease and hypothyroidism in MD varied significantly from 1 to $38 \%$. Notable bias was introduced given the lack of standardization of diagnostic criteria across studies. Articles that described autoimmune thyroid-specific antibodies and HLA types also presented inconclusive results. Multiple studies noted a potential etiologic role of hypothy-
\end{abstract}

roidism in $M D$, which was often confounded by thyroxine supplementation. Conclusions: Despite a potential correlation in the medical literature between thyroid disorders and $M D$, there is currently no definitive causal relationship. Although most of the present medical literature focuses on autoimmunity, dysregulated thyroid hormone levels may also be implicated in the association of MD with thyroid disorders.

(c) 2021 S. Karger AG, Basel

\section{Introduction}

Ménière's disease (MD) is an inner ear disorder that manifests as episodic vertigo, fluctuating hearing loss, tinnitus, and aural fullness. Characteristics of MD include a typical age of onset from the fourth to fifth decades, female predominance, and predilection for those of Northern European descent $[1,2]$. Histopathologic confirmation of MD can only be performed postmortem but classically demonstrates endolymphatic hydrops [3].

This paper was presented as a poster presentation at the AAO-HNSF Annual Meeting on October 2019, New Orleans, LA. 
Currently, the precise etiology of the disrupted regulation of endolymphatic fluid remains unknown and may be due to multiple factors including metabolic disorders, hormone dysfunction, psychological factors, and immunologic disease $[4,5]$. Specifically, autoimmunity as a potential causative factor of MD is strongly supported in the medical literature [6-8].

Evidence supporting the role of autoimmunity in $\mathrm{MD}$ patients includes a positive steroid response, serum inner ear antigen antibodies, and circulating immune complexes $[9,10]$. Furthermore, MD has been associated with several autoimmune disorders including rheumatoid arthritis, systemic lupus erythematosus, ankylosing spondylitis, autoimmune hypothyroidism, and psoriasis [11]. Several studies have also proposed a specific relationship between MD and autoimmune thyroid disease (TD) due to a significantly higher prevalence of antithyroid autoantibodies in MD $[12,13]$. However, debate exists as to whether the dysregulated metabolic state in hypothyroid patients serves alone as a separate etiology of MD, unrelated to autoimmunity. Given these prior research efforts, the goal of this work was to perform a systematic review of the medical literature and evaluate evidence on the prevalence of thyroid autoimmunity and the role of dysregulated thyroid hormone levels in MD.

\section{Materials and Methods}

This study was performed using the Preferred Reporting Items for Systematic Reviews and Meta-Analyses (PRISMA) protocol and exempt from Institutional Review Board Review at Penn State Milton S. Hershey Medical Center [14]. Independent searches of PubMed and MEDLINE databases were performed on June 21, 2018 , by 2 separate authors to validate studies which described both thyroid disorders and Ménière's disease using the Boolean method and relevant search term combinations, that is, "thyroid" and "Ménière's disease." All languages were included in the search algorithm, but only English studies were selected for final analysis [15]. Full-length original articles were assessed for data extraction eligibility and screened by 2 independent reviewers. Duplicated articles and abstracts were excluded, along with non-English (4), nonfull text (1), not Ménière's disease (4), and not thyroid disorders (2). A qualitative synthesis of the available studies was employed to integrate study results (Fig. 1).

\section{Results}

\section{Inclusion Criteria and Study Design}

A total of 171 abstracts were identified via the employed search strategy. Based on inclusion and exclusion

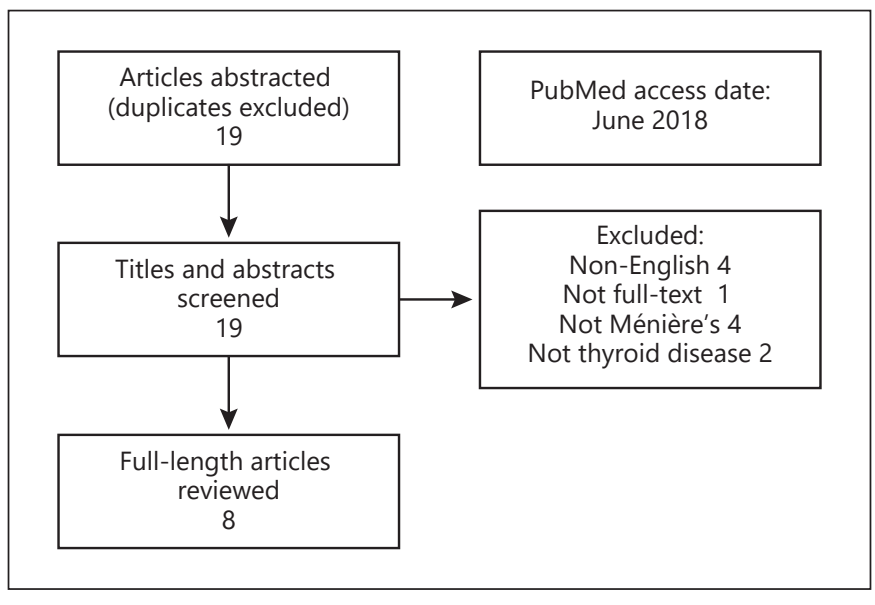

Fig. 1. Search strategy protocol.

criteria, 8 retrospective studies were selected for final analysis [5, 16-18]. Four of 8 studies, including the 3 most recently conducted studies, characterized MD using criteria of AAO-HNS Subcommittee on Equilibrium and its Measurement (Table 1) [5, 19-21]. In addition to using clinical symptomatology, neuro-otological evaluation (sensorineural hearing impairment, SISI score, ABLB, stapedial reflex decay, Bekesy audiometry, tone decay test, and ENG) was performed in half of the studies.

In order to determine the effect of thyroid and allergy treatment on MD symptom control, Powers et al. [5] evaluated $98 \mathrm{MD}$ patients with FTA-ABS, neuro-otological, allergic, and metabolic studies including T4 free index test and T4 serum-bound test. Kinney et al. [15] identified 180 out of 384 total MD patients from May 1976 through May 1979 that had thyroid screening as part of their MD workup. The authors used the classification of Arenberg and Stahle for hearing stage determined by pure-tone average.

Karjalainen et al. [17] compared 91 MD patients to 149 osteosclerotic patients in regard to carbohydrate and lipid metabolism. Meyerhoff et al. [16] retrospectively reviewed 211 patients with MD symptoms in regard to clinical symptomatology, diagnostic testing, and laboratory abnormalities. Both Karjalainen et al. [17] and Meyerhoff et al. [16] used formal audiological and vestibular testing for the diagnosis of MD.

Evans et al. [18] screened serum of 164 patients diagnosed with classic MD, although with unspecified diagnostic criteria, for autoantibodies (thyroglobulin and antithyroid microsomal), immune complexes, and human leukocyte antigen (HLA) status. Brenner et al. [19] performed a retrospective case-control study with $50 \mathrm{MD}$ pa- 


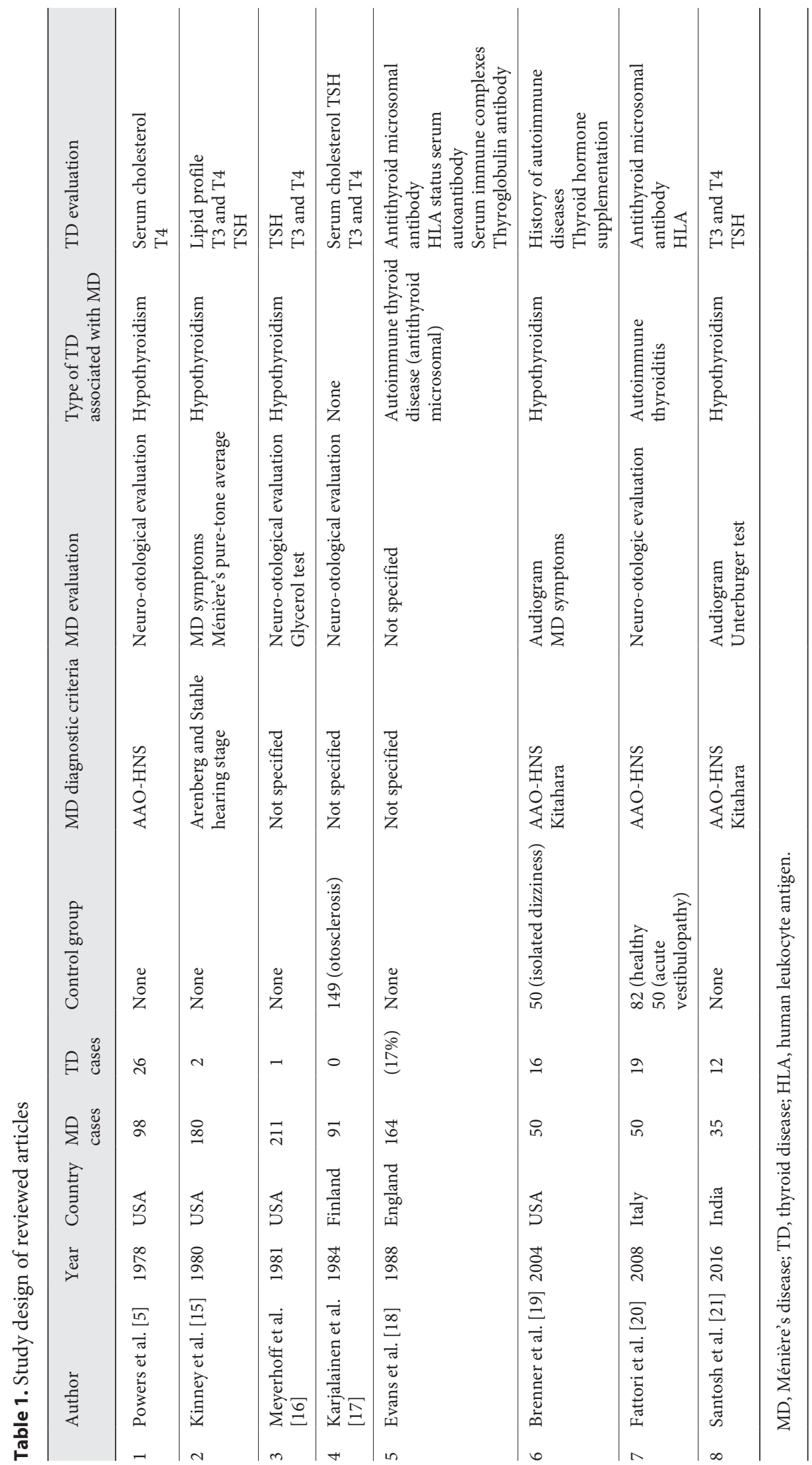


tients and 50 matched control patients. Participants were contacted using phone interviews to evaluate for hypothyroidism or a history of systemic autoimmune disease.

Fattori et al. [20] compared $50 \mathrm{MD}$ patients to 2 matched control groups (healthy individuals and acute unilateral peripheral vestibulopathy patients) and collected HLA and antithyroid titers. Cochlear-vestibular function was assessed with audiometry, and VNG analysis performed during acute attacks of vertigo. Last, Santosh et al. [21] evaluated $35 \mathrm{MD}$ patients using audiogram and assessed the subjective rate of symptom improvement after 3 weeks, 3 months, and 6 months of thyroxine supplementation.

\section{Prevalence of Thyroid Disease in MD}

Of the 8 included studies, 5 studies concluded MD correlated with TD. Powers et al. [5] and Evans et al. [18] both discovered a $17 \%$ prevalence of MD patients associated with nonmyxedematous hypothyroidism and autoimmune TD, respectively (Table 2) $[5,18]$. Brenner et al. [19], Santosh et al. [21], and Fattori et al. [20] reported a prevalence of hypothyroidism and autoimmune thyroiditis ranging from 32 to $38 \%$ [19-21]. Brenner et al. [19] found that $32 \%$ (16 of 50 ) of MD patients were taking thyroid hormone supplementation compared to controls. Among MD patients taking thyroid hormone supplementation versus MD patients not taking thyroid hormone supplementation, Brenner et al. [19] concluded that there was no significant difference in the prevalence, severity, or pattern of hearing loss and found that 4 of 16 patients with corrected hypothyroidism had other systemic autoimmune diseases.

Several studies, however, did not demonstrate this relationship. Kinney et al. [15] found that of 168 patients who underwent a lipid profile, 104 patients (62\%) had elevated cholesterol and/or triglyceride levels, but only 2 MD patients were found to have hypothyroidism. Meyerhoff et al. [16] found that out of 211 MD patients, only 1 patient had abnormal thyroid studies. Finally, Karjalainen et al. [17] demonstrated that of $91 \mathrm{MD}$ patients after a 12 -h fast, $15 \%$ of patients had hyperlipidemia, but 0 patients demonstrated abnormal thyroid studies.

\section{Management: Thyroid Hormone \\ Supplementation}

Multiple studies have noted a potential etiologic role of hypothyroidism in $\mathrm{MD}$, which is often confounded by thyroxine supplementation $[5,19,21]$. Powers et al. [5] found that MD symptoms were controlled with elimination of vertigo and hearing improvement in $3 \%$ of pa- tients with thyroid hormone replacement alone which increased to $14 \%$ in combination with endocrine or allergy treatment for 17 months (Table 2) [5]. Kinney et al. [15] found that dietary management was the most effective method of management in $65 \%$ of MD patients with hyperlipidemia, specifically types II and IV.

Brenner et al. [19] found that 16 of $50 \mathrm{MD}$ patients (32\%) were taking thyroid hormone supplements compared to 2 (4\%) of the 50 controls and that age was directly correlated with $\mathrm{MD}$ patients taking thyroid hormone supplementation. A significant difference was also noted between 5 of $26 \mathrm{MD}$ patients (19\%) younger than 60 years of age taking thyroid hormone supplementation compared to the 11 of 24 patients (46\%) MD patients 60 years of age and older [19].

Santosh et al. [21] identified 12 of $35 \mathrm{MD}$ patients treated with thyroxine supplementation and evaluated symptom improvement at 3 weeks, 3 months, and 6 months. After 12 weeks of treatment, subjective improvement in all symptoms, including vertigo, tinnitus, sensorineural hearing loss, and aural fullness, was appreciated in all cases of hypothyroidism and MD. Elevated TSH levels were also noted in all 12 hypothyroid MD patients who became euthyroid after medical treatment. In addition, mean vertigo score for rotatory sensation, tinnitus, aural fullness, and hearing loss was reduced, and a normal Unterburger test was found in all patients after 12 weeks of thyroid hormone supplementation. The mean improvement in vertigo scores before and after 12 weeks of treatment was a change of 1.42-0.5 for rotation, 1.5-0.5 for tinnitus, $1.33-0.67$ for aural fullness, and $2-1.33$ for hearing loss.

\section{Serological Testing}

Evans et al. [18] and Fattori et al. [20] were the only 2 studies that performed autoimmune serological testing. Evans et al. [18] found that 73 of $150 \mathrm{MD}$ patients had 1 autoantibody, specifically $23.3 \%$ antinuclear, $17 \%$ antithyroid microsomal, and $13 \%$ smooth muscle (Table 2) [18]. Roughly $50 \%$ of patients had more than 1 antibody in both groups. Further testing indicated that $53 \mathrm{MD}$ patients had the HLA Cw7 antigen. Furthermore, the HLA Cw7-positive group had significantly elevated IgM and $\mathrm{C} 1 \mathrm{q}$ levels of immune complexes as well as significantly lower levels of IgA than controls. Overall, increased antithyroid microsomal and antinuclear antibodies were mainly found in MD patients 40-60 years of age.

Fattori et al. [20] found that $38 \%$ of MD patients had elevated serum antithyroid autoantibodies compared to $7 \%$ in healthy controls and $12 \%$ in non-MD vestibulopa- 


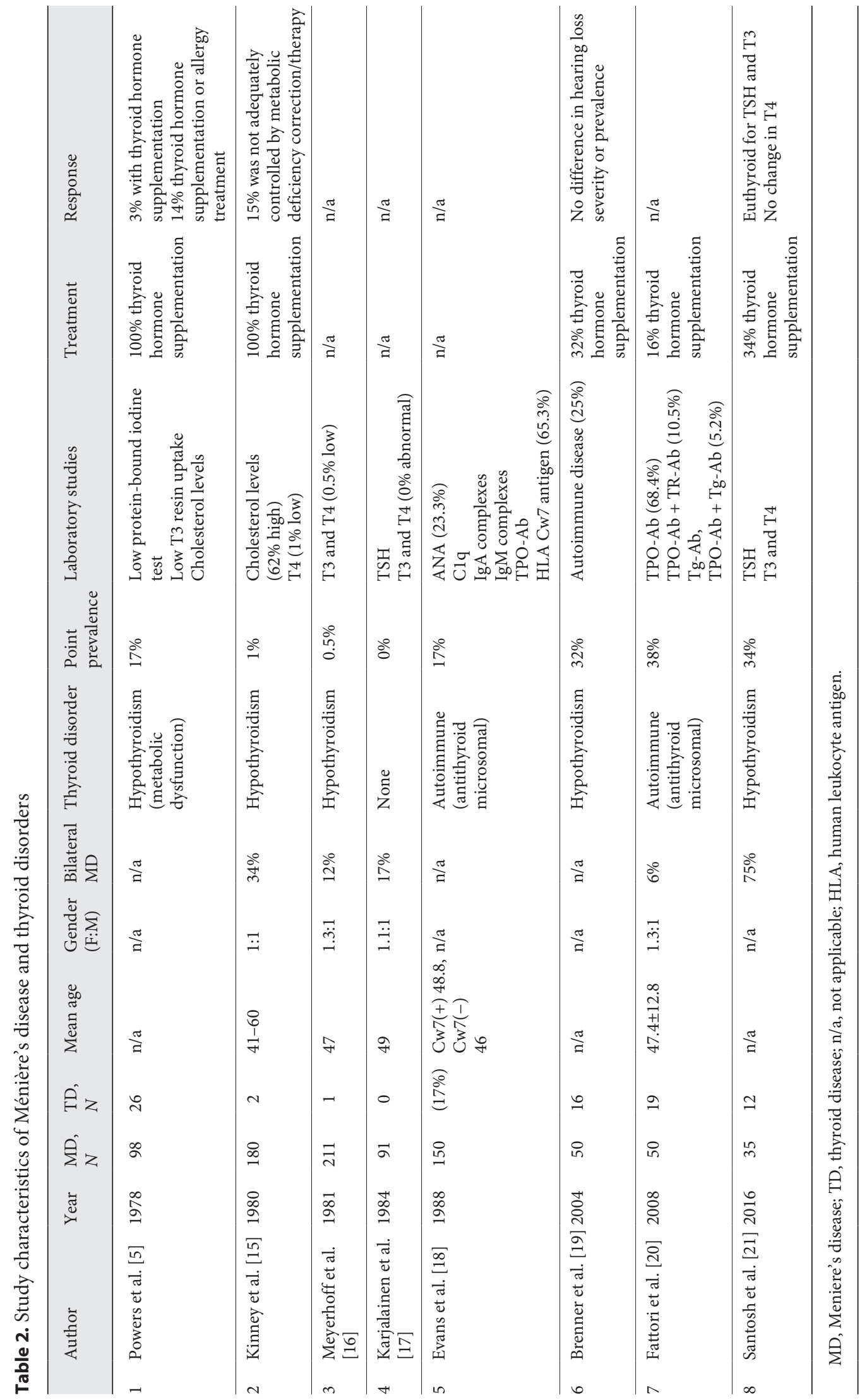


thy (Table 2). Out of the $19 \mathrm{MD}$ patients with high antibody titers, $68.4 \%$ had only thyroid peroxidase antibodies, $10.5 \%$ had only thyroglobulin antibodies, $15.8 \%$ had thyroid receptor antibodies, and 5.2\% had both thyroid peroxidase and thyroglobulin antibodies. Of the $50 \mathrm{MD}$ patients, 8 were previously treated with thyroid supplementation, and 6 of these patients had an elevated antithyroid antibody. Furthermore, there was no relationship between the severity of hearing loss measured by PTA and autoantibody titers, suggesting a lack of an association between overall disease severity of MD and autoimmune thyroiditis.

\section{Discussion}

Out of the 8 included studies, 5 found an association between hypothyroidism and MD [5, 18-21]. Case reports suggesting control of hearing impairment with thyroid hormone supplementation date back to the 1950s [22]. Godlowski et al. [23] first proposed hypometabolism as a potential factor in MD. Spencer et al. [24] concluded that a significantly high incidence of abnormal lipid metabolism occurs in MD. Powers et al. [5] further developed this hypothesis specifying that nonmyxedematous hypothyroidism may be associated with MD along with hyperglycemia, hyperlipidemia, and hypoadrenal dysfunction [15]. Improvement in MD symptoms after thyroid hormone supplementation suggests that thyroid hormone dysfunction and related metabolic disorders may account for a reversible onset of endolymphatic hydrops and a potential subset of MD patients [19, 21, 25, 26].

Conversely, 3 studies did not find a strong association between hypothyroidism and MD, suggesting TD screening and metabolic function tests may not be useful or cost-effective measures in patients without a history specifically concerning for these select disorders [15-17]. Kinney et al. [15] recommended dietary management of hyperlipidemia and abnormal carbohydrate metabolism rather than thyroid hormone replacement due to the higher correlation between MD and hyperlipidemia. Although Kinney et al. [15], Meyerhoff et al. [16], and Karjalainen et al. [17] involved large patient populations ranging from 81 to $180 \mathrm{MD}$ patients, these studies were performed before the discovery of autoimmune thyroid laboratory studies, including autoantibody titers, complement, or immune complexes. Furthermore, some MD patients in these studies were already taking thyroid hormone supplementation and likely achieving a euthyroid state.
Fattori et al. [20] concluded a significant relationship between thyroid autoimmunity and MD, hypothesizing an immune response in the form of autoantibodies against inner ear antigens [10, 27, 28]. Fattori et al. [20] also noted that the pendrin protein associated with Pendred syndrome may be a target for autoantigens as it is expressed in both thyroid and inner ear cells. Hadj et al. [29] further extrapolated and indicated that Pendred syndrome may predispose patients to autoimmune TD. Previous studies have found antibodies in MD against ganglion cells, anticollagen II, and antiphospholipid. Fattori et al. [20] further discussed that pendrin, associated with Pendred syndrome, may be a target for an autoantigen as it is expressed in both the thyroid gland and inner ear. Additionally, Fattori et al. [20] suggested that autoantibodies in MD may predispose patients to other autoimmune diseases and manifest clinically as polyendocrine syndrome including multiple gland autoimmune syndromes [30]. For example, Hashimoto's thyroiditis has been seen in multiple gland autoimmune syndromes such as systemic lupus erythematosus, pernicious anemia, and rheumatoid arthritis [31,32]. A higher prevalence of antithyroid autoantibodies in MD patients than healthy controls has been previously documented.

Evans et al. [18] discussed the possibility of humoral immunity based on increased IgM levels in circulating complexes and high levels of complement. Elevation of these serum proteins is comparable to other autoimmune diseases like systemic lupus erythematosus and polyarteritis nodosa, which can be associated with sensorineural hearing loss and vestibular dysfunction [33, 34]. It has also been hypothesized that inflammatory mediators may lead to capillary vasodilation and complex aggregation in the inner ear capillaries due to the presence of various inner ear antigens such as type II collagen and heat shock protein $70[35,36]$. More recent antibodies studied include MP0-antibodies, antiphospholipid antibodies, and sialyl-I ganglioside antibodies [37-39]. Conflicting studies on the role of circulating immune complexes have indicated further studies are necessary $[40,41]$. With regard to genetics, polymorphic host cell factor 1 gene may affect herpes simplex virus reactivation based on the association between polymorphic host cell factor 1 gene and a reduced risk of MD [42]. MD has also been linked to the PTPN22 gene 1858C/T polymorphism and may play a role in T-cell function [43]. Additionally, symptomatic improvement after oral and intratympanic glucocorticoids in MD suggests an autoimmune relationship; however, these therapies may only be effective in certain MD patients [44]. Although these studies together suggest a 
possible immune etiology of $\mathrm{MD}$, additional research efforts are necessary to determine the role of thyroid autoimmunity in the formation of endolymphatic hydrops.

Notable bias was introduced given that many studies lacked uniform standardization of diagnostic criteria and clinical data reporting. The lack of absolute guidelines for MD makes it challenging to compare studies over time. In addition, methodology between studies, including reporting serologic or metabolic laboratory tests, varied greatly when comparing MD patients to control groups. Thus, given the significant variability in the data collected from each study, meta-analysis was not able to be performed. Furthermore, the low sample size across studies was a consistent limitation of the included articles, which is a challenge when evaluating uncommon clinical disorders like MD.

\section{Conclusion}

It has been suggested that studies correlating autoimmune TD and MD lack replication and consistent identification of a single antibody or HLA complex. Although the studies included in this review suggest a possible autoimmune etiology of $\mathrm{MD}$, additional clinical data are necessary to determine the role of thyroid autoimmunity and hypothyroidism in the development of endolymphatic hydrops. Moreover, further research efforts are needed to validate a common susceptibility factor in MD and autoimmune thyroid disorders and distinguish whether a relationship is secondary to a common immunologic agent or metabolic and hormonal derangements. Thus, it is possible that multiple etiologies of MD could be further classified into different subtypes such as spo- radic, familial, autoimmune, and metabolic. Additional studies are necessary to delineate autoimmune and metabolic relationships in $\mathrm{MD}$; however, it is possible that different etiologies of MD exist and further subtype classification may be necessary.

\section{Acknowledgement}

The authors would like to thank Kimberly Walker, Division of Outcomes Research and Quality, for formatting the manuscript.

\section{Statement of Ethics}

The authors have no ethical conflicts to declare as this is a systematic review.

\section{Conflict of Interest Statement}

The authors have no conflicts of interest to declare.

\section{Funding Sources}

There was no funding for this study.

\section{Author Contributions}

Study concept and design: Patel, Isildak, and Saadi. Acquisition and analysis of data: Patel, Isildak, and Saadi. Interpretation of data: Patel, Liaw, Hwang, Isildak, and Saadi. Drafting of the manuscript: Patel, Liaw, Hwang, Isildak, and Saadi. Critical revision of the manuscript for important intellectual content: Patel, Liaw, Hwang, Isildak, and Saadi. Study supervision: Isildak.

\section{References}

1 Stahle J, Stahle C, Arenberg IK. Incidence of Ménière's disease. Arch Otolaryngol. 1978; 104(2):99-102.

2 Sajjadi H, Paparella MM. Meniere's disease. Lancet. 2008;372(9636):406-14.

3 Hallpike CS, Cairns H. Observations on the pathology of Meniere's syndrome: (section of otology). Proc R Soc Med. 1938;31:1317-36.

4 Baloh RW, Honrubia V, Kerber KA. Baloh and Honrubia's clinical neurophysiology of the vestibular system. Oxford, UK: Oxford University Press; 2013.

5 Powers WH. Metabolic aspects of Meniere's disease. Laryngoscope. 1972;82(9):1716-25.

6 McCabe BF. Autoimmune sensorineural hearing loss. Ann Otol Rhinol Laryngol. 1979 Sep-Oct;88(5 Pt 1):585-9.
7 Hughes GB, Barna BP, Kinney SE, Calabrese LH, Hamid MA, Nalepa NJ. Autoimmune endolymphatic hydrops: five-year review. Otolaryngol Head Neck Surg. 1988;98(3):221-5.

8 Tomiyama S, Harris JP. The endolymphatic sac: its importance in inner ear immune responses. Laryngoscope. 1986;96(6):685-91.

9 Garcia-Purrinos FJ, Ferri E, Rosell A, Calvo J. Combined intratympanic and intravenous dexomethasone to control vertigo in Meniere disease. Acta Otorrinolaringol Esp. 2005;56: 74-7.

10 Yoo TJ, Shea J, Ge X, Kwon SS, Yazawa Y, Sener $\mathrm{O}$, et al. Presence of autoantibodies in the sera of Meniere's disease. Ann Otol Rhinol Laryngol. 2001;110(5 Pt 1):425-9.
11 Jenkins RC, Weetman AP. Disease associations with autoimmune thyroid disease. Thyroid. 2002;12(11):977-88.

12 Tomoda K, Suzuka Y, Iwai H, Yamashita T, Kumazawa T. Meniere's disease and autoimmunity: clinical study and survey. Acta Otolaryngol Suppl. 1993;500:31-4.

13 Morrison AW, Xenellis JD. Immunological aspects of Meniere's disease. Immunobiology, histophysiology and tumour immunology in otolaryngology. Proceedings of the 2nd International Academic Conference Utrecht. Amsterdam: Kugler; 1986. p. 9-14.

14 Moher D, Shamseer L, Clarke M, Ghersi D, Liberati A, Petticrew M, et al. Preferred reporting items for systematic review and metaanalysis protocols (PRISMA-P) 2015 statement. Syst Rev. 2015;4:1. 
15 Kinney SE. The metabolic evaluation in Meniere's disease. Otolaryngol Head Neck Surg. 1979;88(5):594-8.1980

16 Meyerhoff WL, Paparella MM, Gudbrandsson FK. Clinical evaluation of Ménière's disease. Laryngoscope. 1981;91(10):1663-8.

17 Karjalainen S, Nuutinen J, Kärjä J, Vartiainen E. Lack of utility of metabolic screening in Ménière's disease. Clin Otolaryngol Allied Sci. 1984;9(1):15-20.

18 Evans KL, Baldwin DL, Bainbridge D, Morrison AW. Immune status in patients with Menière's disease. Arch Otorhinolaryngol. 1988;245(5):287-92.

19 Brenner M, Hoistad DL, Hain TC. Prevalence of thyroid dysfunction in patients with Ménière's disease. Arch Otolaryngol Head Neck Surg. 2004;130(2):226-8.

20 Fattori B, Nacci A, Dardano A, Dallan I, Grosso $\mathrm{M}$, Traino $\mathrm{C}$, et al. Possible association between thyroid autoimmunity and Menière's disease. Clin Exp Immunol. 2008;152(1):28-32.

21 Santosh UP, Rao MS. Incidence of hypothyroidism in Meniere's disease. J Clin Diagn Res. 2016;10(5):MC01-3.

22 Hilger JA. Otolaryngologic aspects of hypometabolism. Trans Am Laryngol Assoc. 1956; 77:40-62.

23 Godlowski ZZ. Endocrine management of selected cases of allergy based on enzymatic mechanism of sensitization. AMA Arch Otolaryngol. 1960;71:513-57.

24 Spencer JT Jr. Hyperlipoproteinemia and inner ear disease. Otolaryngol Clin North Am. 1975;8(2):483-92.

25 Goebel JA. 2015 equilibrium committee amendment to the 1995 AAO-HNS guidelines for the definition of Ménière's disease. Otolaryngol Head Neck Surg. 2016;154(3) 403-4.
26 Kitahara M. Concepts and diagnostic criteria of Ménière's disease. In: Kitahara M, editor. Ménière's disease. Tokyo: Springer Japan; 1990. p. 3-12.

27 Wei NR, Helms J, Giebel W. Immunohistochemical findings in the vestibular ganglion from a patient with Menière's disease. Eur Arch Otorhinolaryngol. 1990;247(6):340-4.

28 Mouadeb DA, Ruckenstein MJ. Antiphospholipid inner ear syndrome. Laryngoscope. 2005;115(5):879-83.

29 Hadj kacem H, Rebai A, Kaffel N, Masmoudi S, Abid M, Ayadi H. PDS is a new susceptibility gene to autoimmune thyroid diseases: association and linkage study. J Clin Endocrinol Metab. 2003;88(5):2274-80.

30 Barker JM. Polyendocrine autoimmunity. Curr Diab Rep. 2005;5(2):84-90.

31 Chen SH, Hung CS, Yang CP, Lo FS, Hsu HH. Coexistence of megaloblastic anemia and iron deficiency anemia in a young woman with chronic lymphocytic thyroiditis. Int J Hematol. 2006;84(3):238-41.

32 Biró E, Szekanecz Z, Czirják L, Dankó K, Kiss E, Szabó NA, et al. Association of systemic and thyroid autoimmune diseases. Clin Rheumatol. 2006;25(2):240-5.

33 Hamblin TJ, Mufti GJ, Bracewell A. Severe deafness in systemic lupus erythematosus: its immediate relief by plasma exchange. Br Med J. 1982;284(6326):1374.

34 Stephens SD, Luxon L, Hinchcliffe R. Immunological disorders and auditory lesions. Audiology. 1982;21(2):128-48.

35 Plotz PH. Studies on immune complexes. Arthritis Rheum. 1982;25(10):1151-5.

36 Kangasniemi E, Hietikko E. The theory of autoimmunity in Meniere's disease is lacking evidence. Auris Nasus Larynx. 2018;45(3): 399-406.
37 Cao MY, Gersdorff M, Deggouj N, Tomasi JP. The localization and specificity of guinea pig inner ear antigenic epitopes. J Laryngol Otol. 1995;109(1):19-23.

38 Ruckenstein MJ, Prasthoffer A, Bigelow DC Von Feldt JM, Kolasinski SL. Immunologic and serologic testing in patients with Ménière's disease. Otol Neurotol. 2002;23(4): 517-1.

39 Ikeda A, Komatsuzaki A, Kasama T, Handa S, Taki T. Detection of antibody to sialyl-i, a possible antigen in patients with Meniere's disease. Biochim Biophys Acta. 2000;1501(23):81-90.

40 Brookes GB, Newland AC. Plasma exchange in the treatment of immune complex-associated sensorineural deafness. J Laryngol Otol. 1986;100(1):25-33

41 Lopez-Escamez JA, Saenz-Lopez P, Gazquez I, Moreno A, Gonzalez-Oller C, Soto-Varela $\mathrm{A}$, et al. Polymorphisms of CD16A and CD32 $\mathrm{F} c \gamma$ receptors and circulating immune complexes in Ménière's disease: a case-control study. BMC Med Genet. 2011;12:2.

42 Vrabec JT, Liu L, Li B, Leal SM. Sequence variants in host cell factor $\mathrm{C} 1$ are associated with Ménière’s disease. Otol Neurotol. 2008;29(4): 561-6.

43 Lopez-Escamez JA, Saenz-Lopez P, Acosta L, Moreno A, Gazquez I, Perez-Garrigues H, et al. Association of a functional polymorphism of PTPN22 encoding a lymphoid protein phosphatase in bilateral Meniere's disease. Laryngoscope. 2010;120(1):103-7.

44 Phillips JS, Westerberg B. Intratympanic steroids for Ménière's disease or syndrome. Cochrane database Syst Rev. 2011(7):CD008514. 